Objectives Through this case report we aim to present a series of cranial images illustrating the progression of a lytic bone lesion and subsequent infiltration of tortuous arterial vasculature over the course of one year in a preterm neonate with Menkes disease.

Methods Retrospective case review of the management a patient with Menkes disease and a skull fracture over one year.

Results Our patient was born in good condition at $29+5$ weeks gestation via forceps assisted vaginal delivery. There was a family history of maternal carriage of Menkes disease with a second degree relative having had the condition which was the reason for testing in this patient. For treatment of his hypocupremia the patient received intramuscular copper histidine injections, however in the first few weeks of his life serum levels were below the normal range.

Cranial ultrasound scans within the first 7 days of life demonstrated a left sided grade IV intraventricular haemorrhage. $\mathrm{He}$ was noted to have widely splayed sutures with head circumference on the 25th centile. Neurological examination throughout his neonatal admission was normal.

Over time, he developed a persistent boggy swelling over the left parietal region, an ultrasound probe placed over this region revealed an area of absent parietal bone with visualisation of underlying brain parenchyma. A skull x-ray demonstrated a left parietal skull fracture which was further demonstrated on CT scan. This confirmed a mid-left parietal fracture which extended posteriorly from the left coronal suture with a protruding soft tissue component.

Interestingly, the patient had previously had a cranial MRI performed as part of his diagnosis for Menkes disease. Retrospectively a protrusion of subarachnoid space in the left parietal region was seen which was not appreciated initially. $\mathrm{He}$ was transferred to a tertiary hospital for neurosurgical review and subsequently managed conservatively.

Over the course of a year there were neurological and respiratory deterioration secondary to his Menkes disease despite copper histidine injections, necessitating admission to paediatric intensive care. MRI scans performed during this time found an evolution of abnormalities in the left parietal area with diffuse arterial tortuosity and a left parietal pseudomeningocele. The patient passed away during his intensive care admission aged under 1 year.

Conclusions This case illustrated the evolution of a lytic parietal bone lesion with the progression of arterial tortuosity. Recent literature has described cases studies of neonatal skull fracture as a known presentation of Menkes disease. However, with this case study we demonstrate the changes of both a skull lytic lesion in combination with arterial tortuosity as a series of images over time from a single patient. We hope through this case to increase knowledge and awareness in recognising these changes in the context of a patient with Menkes disease.

\section{PROLIDASE DEFICIENCY IN AN INFANT WITH AN INCIDENTAL FINDING OF METHAEMOGLOBINAEMIA}

Chern Tan, Easwari Kothandaraman, Arunabha Ghosh.

10.1136/bmjpo-2021-RCPCH.90

Background A 4-week-old infant presented to hospital with diarrhea and vomiting. An initial diagnosis of cow's milk allergy was made and he was discharged with extensively hydrolysed formula. However the infant was readmitted within a week with ongoing symptoms and associated metabolic acidosis. He was suspected to have sepsis and treated with intravenous antibiotics. However, he deteriorated further with worsening metabolic acidosis despite treatment. Methaemoglobinaemia was then identified and he was treated with methylene blue and transferred to HDU for further management.

\section{Objectives}

1. Consider Methaemoglobinaemia as a differential diagnosis in infants with unexplained metabolic acidosis.

2. Raise awareness on Prolidase deficiency - a rare genetic condition affecting 1 in 1 million of worldwide population.

Methods Literature search on Ovid using Medline/Embase was undertaken.

\section{Results}

Conclusions Further investigations results of Imidopeptiduria and rapid exome sequencing confirmed diagnosis of Prolidase deficiency (PD) in this infant. PD is a rare autosomal recessive genetic condition caused by mutations in PEPD gene, which codes for Prolidase, an enzyme involved in the final stage of the degradation of collagen and other proline containing proteins including dietary proteins. PD affects approximately 1 in 1 million worldwide. The symptoms of PD include dysmorphic features, skin lesions, recurrent infections, hepatosplenomegaly and intellectual disability.

In this case, the child had symptoms of diarrhoea, likely secondary to $\mathrm{PD}$. As the diarrhoea was persistent, he then

\begin{tabular}{ll} 
Abstract 165 Table 1 & \\
\hline Blood gas pre-methylene blue & Blood gas post-methylene blue \\
\hline pH 7.18 & pH 7.30 \\
pCO2 3.9 & pCO2 4.0 \\
HCO3 10.8 & HCO3 19 \\
Lactate 5.7 & Lactate 2.0 \\
MetHb $>30 \%$ & MetHb $7 \%$ \\
\hline
\end{tabular}

\begin{tabular}{|c|c|}
\hline $\begin{array}{l}\text { Full Blood Count( FBC) } \\
\text { (on admission) }\end{array}$ & $\begin{array}{l}\text { Hb } 120 \mathrm{~g} / \mathrm{l} \text {, WCC } 18.6 \times 10^{9} / \mathrm{l} \text {, Neutrophils } 11.69 \times 10^{9} / \mathrm{l} \\
\text { Platelets } 544 \times 10^{9} / \mathrm{l}\end{array}$ \\
\hline $\begin{array}{l}\text { FBC (on day of } \\
\text { deterioration) }\end{array}$ & $\begin{array}{l}\text { Hb } 125 \mathrm{~g} / \mathrm{l} \text {, WCC } 40.7 \times 10^{9} / \text {, Neutrophils } 39 \times 10^{9} / \text {, } \\
\text { Platelets } 653 \times 10^{9} / \mathrm{l}\end{array}$ \\
\hline CRP & $31 \mathrm{mg} / \mathrm{l}$ (on admission), increased to 75 \\
\hline Urea \& Electrolytes & $\begin{array}{l}\text { Na } 133 \mathrm{mmol} / \mathrm{l}, \mathrm{K} 4.1 \mathrm{mmol} / \mathrm{l} \text {, Urea } 0.9 \mathrm{mmol} / \mathrm{l} \text {, Creatinine } 17 \\
\text { umol// }\end{array}$ \\
\hline Blood culture & No growth after 5 days \\
\hline Ammonia & $36 \mathrm{umol} / \mathrm{l}$ \\
\hline Plasma amino acid & $\begin{array}{l}\text { Normal level of alanine, tyrosine, phenylalanine, lysine, } \\
\text { ornithine }\end{array}$ \\
\hline Cerebrospinal fluid & $\begin{array}{l}\text { Culture -no growth, WCC } 23, \mathrm{RBC}<3 \text {, negative } \\
\text { meningococcal/pneumococcal } P C R \text {, negative virology }\end{array}$ \\
\hline Urine culture & No growth \\
\hline Urine amino acids & Imidopeptiduria \\
\hline $\begin{array}{l}\text { Rapid trio exome } \\
\text { sequencing }\end{array}$ & $\begin{array}{l}\text { Homozygous pathogenic PEPD variant }\{c .978 \mathrm{G}>\mathrm{A}, \mathrm{p} \text {. } \\
(\text { (Trp326*)\}. }\end{array}$ \\
\hline
\end{tabular}


developed methaemoglobinaemia as a result. Initial methaemoglobin was $48 \%$ which subsequently improved to $3 \%$ following 3 doses of methylene blue with good clinical response. Further investigations excluded Systemic lupus erythematosus and Crohn's disease, which have been reported as associated with PD. The infant's recovery was supported with parental nutrition and discharged home with ongoing follow up with Paediatric metabolic specialists.

Management of PD is mainly supportive with consideration of use of co-factors that can improve collagen stability, suppression of collagenase and topical application of ointments containing L-proline. Enzyme replacement is also being actively researched.

This case illustrates the importance of prompt recognition and treatment of methaemoglobinaemia in infants with unexplained metabolic acidosis. It also highlights awareness of PD, which although, is extremely rare, can be diagnosed through metabolic screening tests and genetic testing, thereby leading to earlier supportive management.

\section{DEVELOPMENT OF AN ACUTE GENERAL PAEDIATRICS SERVICE SPECIFICATION AT A MAJOR LONDON TEACHING HOSPITAL}

Julia Avery, Navindi Fernandopulle. UK

\subsection{6/bmipo-2021-RCPCH.91}

Background Acute general paediatrics is responsible for the care of children (0-18) presenting acutely to hospital, usually through either the emergency department (ED) or following a GP consultation. In the acute setting patients may be looked after in the emergency department, paediatric assessment units, ambulatory or day care facilities, inpatient wards, operating theatres, intensive care units and outpatient clinics. In a teaching hospital setting, general paediatricians may also have a role co-ordinating care for complex children.

In the last five-ten years, standards from national and regional bodies govern the care provided to patients by general paediatricians. Services and individual practitioners can be called upon to demonstrate how they meet these standards and evidence the quality of care provided, such as at CQC inspection. Relevant documents, however, may be located in multiple places and are not always easily accessible to staff or adequately monitored as part of service governance.

Objectives

- To derive a practitioner focused service specification based on relevant acute paediatric care standards

- To determine local compliance against standards and identify measures needed to achieve compliance

- To plan a robust, practitioner-led methodology for ensuring ongoing compliance and monitoring

Methods Relevant guidance was identified through a variety of search methods: national bodies' websites, expert opinion and liaison with Trust managers to identify standards reported to regulators.

Standards were collated and reviewed amalgamating any areas of duplication and removing those not relevant. The final list was thematically analysed into key areas of practice. An expert group consensus methodology was then used to rate the service performance against these standards.
Results 65 standards of care were identified from six key documents. Thematic analysis identified nine main themes and 16 subthemes.

Using a Red, Amber, Green (RAG) rating system to identify the services' performance against each theme, an action plan was then created, using a plan, do, study, act (PDSA) process to achieve these standards that were then linked to the service governance strategy and to consultant action plans.

It was decided to audit one of the standards with an amber status to assess if it were being met and, if not, why not. There was a $66.6 \%$ compliance, monitored across three paediatric wards, and five themes were identified as reasons for it not being met.

Following on from the data collected, further standards not being met will be explored and their Action Plans will continue to be put into place with their progress monitored regularly within departmental governance meetings.

Conclusions The new service specification is used by clinical staff to reflect on practice and outcomes, allowing easy access to relevant standards to guide clinical practice and service development and quality improvement in a strategic and co-ordinated way. The document is a 'living' document, accessible to all clinical team members and external stakeholders.

It will aid planning, commissioning and provision of acute paediatric services and provide a framework against which to audit provision and demonstrate improvement. A similar methodology could be used across the UK.

\section{USING NETWORK ANALYSIS TO FOLLOW MENINGOCOCCAL SEPTIC SHOCK PATHOGENESIS IN INFANTS}

Javed Ismail, Govind Benakatti, Mohammed Uddin, Asrar Rashid. United Arab Emirates

\subsection{6/bmjpo-2021-RCPCH.92}

Background Previously we reported the complex nature of the transcriptome in Meningococcal sepsis (MenS) limiting the detection of a representative biomarker from a single time point for a dynamic disease process [1]. In this study, alleviating that limitation, we utilised network methodology to the transcriptome of MenS patients obtained from multiple time points during disease progression.

\section{Objectives}

Methods We applied weighted gene co expression network analysis (WGCNA) [2] on normalized expression data of 5 children (P1-P5) with MenS (4 confirmed and 1 clinically assumed) who had blood samples taken at admission (designated 0 hours), 4, 8, 12 and 48 hours (T1-T5). The patients had no previous, comorbidities. We extracted RNA from blood and then the samples were checked by capillary electrophoresis and spectrophotometry for quality control purposes. We conducted microarray (Human Gene 1.0 ST Arrays with 33,297 probes) gene expression experiments to capture the transcriptome profile at each time point.

Results Our initial differential gene analysis between all-time points against T1 showed significant $(\mathrm{p}<1.0 \times 10-7)$ up regulation of protein binding and immune response activities. Cluster map was compiled (figure 1) and then this was matched to trait data (figure 2). (Next, applying WGCNA analysis we identified 18 cluster (or modules) that have distinct topological characteristics (sizes ranging from 